\title{
Heavy quarkonia in a medium as a quantum dissipative system: Master-equation approach
}

\author{
Nicolas Borghini and Clément Gombeaud \\ Fakultät für Physik, Universität Bielefeld, Postfach 100131, D-33501 Bielefeld, Germany
}

(Dated: August 25, 2018)

\begin{abstract}
The problem of the evolution of a heavy quarkonium in a medium can be recast as that of a quantum dissipative system. Within the framework of the master-equation approach to open quantum systems, we consider the real-time dynamics of quarkonia. We find that in a plasma at fixed temperature, the populations of the various quarkonium states evolve together, while their momentum distribution satisfies a Fokker-Planck equation.
\end{abstract}

PACS numbers: 25.75.Nq, 12.38.Mh, 14.40.Pq

\section{INTRODUCTION}

The original idea that heavy quarkonia might be suppressed in a deconfined QCD medium, thereby probing the formation of a quark-gluon plasma in high-energy nuclear collisions 1], has motivated many studies (see Refs. 2[4] for recent reviews). The properties of quarkonia in a medium - be it deconfined or hadronic, as it was realized that the most fragile states might already be destroyed in a hot hadron gas - have been extensively investigated, both in lattice QCD calculations and using effective field-theoretical approaches [5].

In particular, it was understood that a description of heavy quarkonia in a medium as non-relativistic quarkantiquark systems bound by an effective static potential is possible, provided the potential has an imaginary part [6 11], which accounts for the finite lifetime of the states. This emphasizes the necessity to consider the real-time dynamics of quarkonia, which becomes even more pregnant when the medium is rapidly expanding and cooling down, as is the case of the fireball in nucleusnucleus collisions.

For that purpose, it is interesting to consider alternative modelings of the influence of the medium on the embedded $Q \bar{Q}$ pair. Noting that the latter is a "small system", then the surrounding medium can be seen as a "reservoir", which can exchange energy and momentum with the small system without being noticeably affected. This is analogous to the paradigm setup for quantum dissipative systems [12], which suggests to view a quarkonium in a medium as such an open quantum system 13, 14].

Accordingly, it becomes natural to study the dynamical evolution of $Q \bar{Q}$ pairs in a medium with the help of the techniques developed in the context of quantum dissipative systems. In a forthcoming paper 15], we shall consider a wavefunction-based approach to obtain the dynamics of quarkonia. Here, we use the master-equation formalism, and derive the time evolution of the populations of $Q \bar{Q}$ states [14], as well as of the momentum distribution of the quarkonia. For the latter, we show that it satisfies a Fokker-Planck equation, with transport coefficients fixed by the microscopic model.
Various kinetic frameworks for the dynamics of quarkonia in a medium have been considered in the literature, based on the Boltzmann [16-18], Fokker-Planck or Langevin equations [19, 20] or rate equations [21, 22], to model the destruction and (re-)formation of bound $Q \bar{Q}$ states in phenomenology-oriented studies. Here the open-quantum-system approach to heavy quarkonia in a medium provides a natural underlying microscopic description that leads to such a kinetic model.

In Sect. II we introduce the general theoretical framework that we shall afterwards apply to obtain a microscopic description of the in-medium evolution of quarkonia. Section III] introduces our model for the heavy quarkonia, the medium, and their interaction between them. Since we intend the present study to be of exploratory nature, we deliberately adopt a simplified model, instead of more realistic ones. Our results for the evolution of both the internal and external degrees of freedom, namely the populations of the various states and the $Q \bar{Q}$-pair momentum distribution function respectively, are presented in Sect. IV] Finally, we discuss our model together with the underlying assumptions and our results in Sect. V], where we also consider how these results might be modified in an evolving medium.

\section{EVOLUTION EQUATIONS}

In this section, we briefly review the master-equation description of quantum dissipative systems for the sake of self-containedness (a longer presentation can be found e.g. in Ref. [23]). After introducing in Sect. IIA the generic setup and its description, we present the equations that govern the evolution of the dissipative system, starting with its internal degrees of freedom (Sect. IIB) and then turning to the external ones (Sect. IC).

\section{A. Small quantum system coupled to a reservoir}

Generically, the setup for a quantum dissipative system consists of a (small) system $\mathcal{S}$ coupled to another quantum system $\mathcal{R}$, called environment - or reservoir, if 
it has infinitely many degrees of freedom, as we shall assume from now on. The total system $\mathcal{S}+\mathcal{R}$ is assumed to be closed. It is then described by a Hamiltonian, taken to be of the form

$$
H=H_{\mathcal{S}}+H_{\mathcal{R}}+V
$$

where $H_{\mathcal{S}}$ denotes the free Hamiltonian of the small system (in the absence of the environment), $H_{\mathcal{R}}$ is the free Hamiltonian of the reservoir, and $V$ describes the interaction between system and environment.

Hereafter, we shall model the reservoir as a set of harmonic oscillators, labeled by a subscript $\lambda$, whose proper frequencies $\omega_{\lambda}$ span a large continuum, encompassing the Bohr frequencies of the free Hamiltonian $H_{\mathcal{S}}$. Let $\rho^{\mathcal{R}}$ denote the density operator of the free reservoir.

For the interaction term in the Hamiltonian, we consider a coupling of the form

$$
V=S R \quad \text { with } \quad R=\sum_{\lambda}\left(g_{\lambda} a_{\lambda}+g_{\lambda}^{*} a_{\lambda}^{\dagger}\right)
$$

where $S$ acts on $\mathcal{S}$ only, while $a_{\lambda}$ and $a_{\lambda}^{\dagger}$ are the annihilation and creation operators for oscillator $\lambda$, and $g_{\lambda}$ measures the corresponding coupling.

For a large reservoir, the autocorrelation function $\langle R(t) R(t-\tau)\rangle$ takes non-negligible values only in a small interval around $\tau=0$, of typical size $\tau_{c}$.

Evolution equations for quantities pertaining to the small system are conveniently obtained by introducing first the density operator $\rho$ of the total system, whose evolution is then governed by Heisenberg's equation with the Hamiltonian (11). Iterating the latter (in the Dirac interaction picture) and performing a partial trace over the reservoir degrees of freedom, one finds an exact, yet non-local in time, evolution equation for the "reduced" density operator

$$
\rho^{\mathcal{S}}(t) \equiv \operatorname{Tr}_{\mathcal{R}}(\rho(t))
$$

To obtain more tractable equations, a few simplifying hypotheses are needed. The first one consists of assuming that the total density operator is factorizable at any time:

$$
\rho(t) \simeq \rho^{\mathcal{S}}(t) \otimes \rho^{\mathcal{R}}, \quad \forall t .
$$

This amounts on the one hand to neglecting correlations between the small system and the reservoir beyond a certain order in the interaction term - typically, beyond second order. On the other hand, keeping the free density operator $\rho^{\mathcal{R}}$ in the presence of an interaction with $\mathcal{S}$ amounts to assuming that the latter does not modify the reservoir properties, which is reasonable for the application we have in mind.

The second assumption is that the typical time scale for the evolution of the small system should be much larger than the typical time scale $\tau_{c}$ of the reservoir fluctuations.

Under these two hypotheses, one can derive a firstorder differential "master" equation for the reduced density operator $\rho^{\mathcal{S}}(t)$ [23].

\section{B. Evolution of the internal degrees of freedom}

In a first step, one can focus on the evolution of the internal degrees of freedom of the small system, momentarily leaving aside its motion.

Let $|i\rangle,|j\rangle \ldots$ denote the eigenstates of the free Hamiltonian $H_{\mathcal{S}}$, with $E_{i}, E_{j} \ldots$ the corresponding energies. In the basis spanned by $|i\rangle\langle i|| j\rangle,\langle j| \ldots$, the elements of the reduced density operator obey a set of coupled firstorder differential equations with constant coefficients.

For our purpose, it is sufficient to consider the diagonal elements $\rho_{i i}^{\mathcal{S}}$, corresponding to the populations of the energy eigenstates. These populations satisfy coupled Einstein equations of the form

$$
\frac{\mathrm{d} \rho_{i i}^{\mathcal{S}}}{\mathrm{d} t}(t)=-\sum_{k \neq i} \Gamma_{i \rightarrow k} \rho_{i i}^{\mathcal{S}}(t)+\sum_{k \neq i} \Gamma_{k \rightarrow i} \rho_{k k}^{\mathcal{S}}(t),
$$

with transition rates given (when considering the master equation up to second order in the interaction term) by Fermi's golden rule. For $E_{k}>E_{i}$ and $\omega_{k i} \equiv\left(E_{k}-E_{i}\right) / \hbar$ the corresponding Bohr frequency, one easily finds

$$
\begin{aligned}
\Gamma_{k \rightarrow i} & =\frac{2 \pi}{\hbar^{2}} \sum_{\lambda}\left(\left\langle n_{\lambda}\right\rangle+1\right)\left|\left\langle i ; 1_{\lambda}|V| k ; 0\right\rangle\right|^{2} \delta\left(\omega_{\lambda}-\omega_{k i}\right), \\
\Gamma_{i \rightarrow k} & =\frac{2 \pi}{\hbar^{2}} \sum_{\lambda}\left\langle n_{\lambda}\right\rangle\left|\left\langle k ; 0|V| i ; 1_{\lambda}\right\rangle\right|^{2} \delta\left(\omega_{\lambda}-\omega_{k i}\right),
\end{aligned}
$$

where $\left\langle n_{\lambda}\right\rangle$ denotes the average number of excitations in mode $\lambda$. These rates obviously correspond to emission (with the +1 term accounting for spontaneous emission) and absorption, respectively.

To exemplify the behavior of populations described by Eqs. (5)-(6), we show in Fig. 10 the time dependence of the populations of a 4 -level system $\mathcal{S}$, initially in its ground state, in contact with a thermal bath at temperature $T$. In that case, $\left\langle n_{\lambda}\right\rangle$ is given by the average occupation

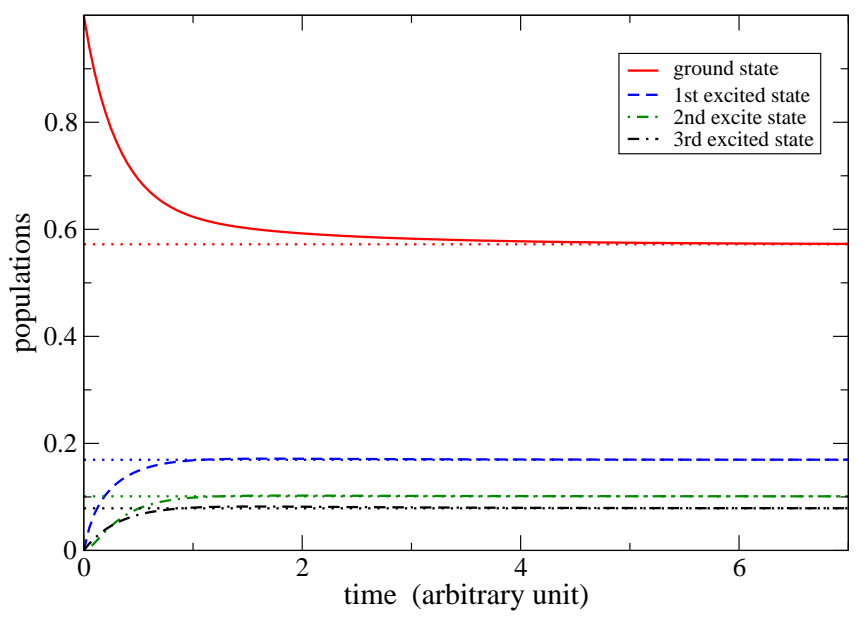

FIG. 1. Time dependence of the populations of the states of a 4-level system coupled to a thermal bath. The straight lines correspond to the equilibrium values at the bath temperature. 
number for Bose-Einstein statistics. After a transient regime, the populations reach stationary values, which are simply proportional to the corresponding Boltzmann factors:

$$
\left(\frac{\rho_{k k}^{\mathcal{S}}}{\rho_{i i}^{\mathcal{S}}}\right)_{\mathrm{eq} .}=\exp \left(-\frac{E_{k}-E_{i}}{k_{B} T}\right) .
$$

We thus find that the internal degrees of freedom of the small system equilibrate at temperature $T$.

\section{Evolution of the external degrees of freedom}

If we also consider the motion of the center of mass of the small system, then momentum transfers due to emission or absorption of excitations from the reservoir now play a role. In addition, the dependence of the interaction term (2) on the position $\mathbf{X}$ of the small system should now be taken into account. For the case we shall be interested in later on, this can be done by replacing $a_{\lambda}$ by $a_{\lambda} \mathrm{e}^{\mathrm{i} \mathbf{k}_{\lambda} \cdot \mathbf{X}}$ with $\left|\mathbf{k}_{\lambda}\right|=\omega_{\lambda} / c$.

The main difference with the static case is that the eigenstates of the free Hamiltonian $H_{\mathcal{S}}$ are states with not only different internal quantum numbers, but also different momenta, corresponding to the $\mathbf{P}^{2} / 2 M_{\mathcal{S}}$ part of $H_{\mathcal{S}}$, with $M_{\mathcal{S}}$ the mass of the small system and $\mathbf{P}$ its total-momentum operator. Consequently, the eigenstates should be labeled with the eigenvalue $\mathbf{p}$ besides the "internal" label $i$.

Introducing now the shorthand notation $\pi_{i, \mathbf{p}} \equiv \rho_{i i, \mathbf{p} p}^{\mathcal{S}}$ for the diagonal terms of the reduced density matrixwhich can be viewed as momentum distributions when they are considered as functions of $\mathbf{p}$ - the evolution equations (5) become

$$
\frac{\mathrm{d} \pi_{i, \mathbf{p}}}{\mathrm{d} t}(t)=\sum_{k \neq i ; \mathbf{q}}\left[\Gamma_{k, \mathbf{q} \rightarrow i, \mathbf{p}} \pi_{k, \mathbf{q}}(t)-\Gamma_{i, \mathbf{p} \rightarrow k, \mathbf{q}} \pi_{i, \mathbf{p}}(t)\right],
$$

with rates again given by Fermi's golden rule. In evaluating the latter, it is convenient to consider the positiondependent part of the interaction Hamiltonian apart from the rest. This part, convoluted with the positiondependent part of the $H_{\mathcal{S}}$ eigenstates - namely plane waves - gives rise to a momentum-conservation enforcing term $\delta_{\mathbf{p}, \mathbf{q}-\hbar \mathbf{k}_{\lambda}}$ in the matrix element $\left\langle k, \mathbf{q} ; 0|V| i, \mathbf{p} ; 1_{\lambda}\right\rangle$. Besides, one should also include the kinetic energy contributions. All in all, one finds for the transition rates between two levels with $E_{k}>E_{i}$

$$
\begin{aligned}
\Gamma_{i, \mathbf{p} \rightarrow k, \mathbf{q}}= & \frac{2 \pi}{\hbar^{2}} \sum_{\lambda}\left\langle n_{\lambda}\right\rangle\left|\left\langle k ; 0|\tilde{V}| i ; 1_{\lambda}\right\rangle\right|^{2} \\
& \times \delta_{\mathbf{p}, \mathbf{q}-\hbar \mathbf{k}_{\lambda}} \delta\left(\omega_{\lambda}-\omega_{k i}-\xi_{D}+\xi_{R}\right), \\
\Gamma_{k, \mathbf{q} \rightarrow i, \mathbf{p}}= & \frac{2 \pi}{\hbar^{2}} \sum_{\lambda}\left(\left\langle n_{\lambda}\right\rangle+1\right)\left|\left\langle i ; 1_{\lambda}|\tilde{V}| k ; 0\right\rangle\right|^{2} \\
& \times \delta_{\mathbf{p}, \mathbf{q}-\hbar \mathbf{k}_{\lambda}} \delta\left(\omega_{\lambda}-\omega_{k i}-\xi_{D}-\xi_{R}\right),
\end{aligned}
$$

where $\tilde{V}$ denotes the position-independent part of the interaction term. $\xi_{D}$ and $\xi_{R}$ are the frequency shifts due to the Doppler effect and the recoil effect, respectively:

$$
\xi_{D} \equiv \frac{\mathbf{k}_{\lambda} \cdot \mathbf{p}}{M_{\mathcal{S}}}-\frac{\omega_{\lambda}}{2} \frac{\mathbf{p}^{2}}{M_{\mathcal{S}}^{2} c^{2}}, \quad \xi_{R} \equiv \frac{\hbar \mathbf{k}_{\lambda}^{2}}{2 M_{\mathcal{S}}},
$$

where the Doppler effect has been considered up to second order. Inserting the rates (8) into Eqs. (7), the sums over $\mathbf{q}$ disappear thanks to the momentum-conservation condition.

Further analytical progress with the population evolution equations requires additional assumptions, namely first that the frequency shifts $\xi_{D}, \xi_{R}$ remain much smaller than the typical width $\Delta \omega$ of the bath spectral distribution; and, secondly, that the momentum transfer $\hbar k_{\lambda}$ be much smaller than the width $\Delta p$ of the populations $\pi_{i, \mathbf{p}}$, viewed as momentum distributions.

Let the sum of the populations $\pi_{i, \mathbf{p}}$ over all internal states $i$ be denoted by $\pi(\mathbf{p})$, which then represents the momentum distribution function of the small system, irrespective of its internal state. As shown in Appendix A3, under the assumptions mentioned above the rate of evolution for $\pi(\mathbf{p}, t)$ is much slower than the individual rates $\mathrm{d} \pi_{i, \mathbf{p}} / \mathrm{d} t$, and one can show that $\pi(\mathbf{p}, t)$ is governed by

$$
\frac{\partial \pi(\mathbf{p}, t)}{\partial t}=\eta_{D} \nabla_{\mathbf{p}} \cdot[\mathbf{p} \pi(\mathbf{p}, t)]+\kappa \triangle_{\mathbf{p}} \pi(\mathbf{p}, t),
$$

i.e. an equation of the Fokker-Planck type, in momentum space, with constant coefficients $\eta_{D}$ and $\kappa$. The former describes the damping rate of both the average momentum and (up to a factor of 2) the variance of the momentum distribution, while the latter characterizes the growth of this variance. When the reservoir in which the small system evolves is a thermal bath, both coefficients are related to each other through the fluctuationdissipation relation

$$
\frac{\kappa}{M_{\mathcal{S}}}=\eta_{D} k_{B} T
$$

which shows that in the stationary regime, the small system has thermalized at the bath temperature $T$.

\section{MODELING QUARKONIUM AS A QUANTUM DISSIPATIVE SYSTEM}

As stated in the introduction, our goal in the present paper is not to propose a full treatment of the dynamics of heavy quarkonia in a thermalized medium based on the most refined existing models for quarkonia and their interaction with the medium. Our purpose is rather to explore possible new qualitative phenomena, which emerge when the point of view on the problem is shifted from the usual approaches to the description as a quantum dissipative system [14].

For that reason, the models we introduce hereafter for the quarkonia (Sect. IIIB) and their coupling to the 
medium (Sect. IIIC) will be quite simplified, yet not unrealistically. For the "reservoir" with which the $Q \bar{Q}$ states interact, we consider two possibilities: either a thermal bath, or a peaked distribution (Sect. IIIA).

\section{A. Medium as a reservoir}

In nuclear collisions at sufficiently high energies, the medium which is created and which is probed by heavy quarkonia should be deconfined, and thus consist of quarks and gluons as relevant degrees of freedom. For the sake of simplicity, we consider a medium made of pure glue, and forget the constantly created quark-antiquark pairs, which would not affect the qualitative features of our description. We assume that this gluon plasma is unpolarized, isotropic and homogeneous.

This plasma can then be modeled-for example by quantizing the $S U(3)$ gauge fields canonically in the Weyl gauge - as a set of oscillators. Within the masterequation formalism, the only characteristic we need is the mean number of excitations $\left\langle n_{\lambda}\right\rangle$ for each mode $\lambda$, see the transition rates (6) and (8).

In the following, we shall make use of two different kinds of gluon bath. First, we shall consider a thermal bath, i.e. a reservoir in thermal equilibrium, at a temperature $T$. The associated density operator reads

$$
\rho^{\mathcal{R}}=\frac{\mathrm{e}^{-H_{\mathcal{R}} / k_{B} T}}{\operatorname{Tr}\left(\mathrm{e}^{-H_{\mathcal{R}} / k_{B} T}\right)}
$$

in the absence of the small system. The corresponding $\left\langle n_{\lambda}\right\rangle$ is given by the usual Bose-Einstein distribution.

The second model of reservoir we shall employ consists of assuming a Gaussian distribution

$$
\left\langle n_{\lambda}\right\rangle \propto \exp \left[-\left(\frac{\hbar \omega_{\lambda}-\bar{E}}{2 \Delta E}\right)^{2}\right],
$$

peaked around some variable value $\bar{E}$, for the average number of excitations. Although this is of less immediate relevance for the phenomenology of ultrarelativistic heavy-ion collisions than the thermal bath, yet it will allow us to illustrate some features of our description. This second model will be referred to as the "Gaussian bath".

\section{B. $Q \bar{Q}$ states}

Strictly speaking, to implement the master-equation formalism described in Sect. III one only needs matrix elements for reservoir-induced transitions between states of the small system. Specifying the states themselves and the transition-inducing interaction is not necessary.

Accordingly, for the study of the dynamics of heavy quarkonia in a gluon plasma, one should identify all single- or multi-gluon processes that change the state of a $Q \bar{Q}$ pair-be it a transition between two different bound states [24], gluon-induced dissociation [25, 26], or the possible recombination of a quark and an antiquark into a bound state [27] - , and consider the corresponding matrix elements. Such an exhaustive procedure is certainly desirable for making quantitative predictions that can meaningfully be compared to experimental results. Here we remain at an exploratory level, and search the qualitative behaviors of quarkonia in a medium. To make amend for our not using the most accurate set of matrix elements, we do not restrict ourselves to postulating such a set, but we shall start from scratch, i.e. from a model of quarkonia in the vacuum, and of their interaction with the gluon plasma introduced above.

For the purpose of identifying new phenomena, the bottomonium system, with its larger number of bound states likely to survive above the deconfinement temperature [5], seems to us more promising than the charmonia. A further advantage of bottomonia, is that (in vacuum) they can reasonably be described as bound energy eigenstates, with simple wavefunctions, of a one-gluonexchange Coulomb potential

$$
V_{Q \bar{Q}}(r)=-C_{F} \frac{\alpha_{s} \hbar c}{r}
$$

with $C_{F}=4 / 3$ the usual color factor and $\alpha_{s}$ the dimensionless (running) coupling constant, here $\alpha_{s} \simeq 0.25$. For charmonia, this would be a less satisfactory description.

There are several drawbacks to our modeling bottomonia as $b \bar{b}$ pairs bound by a Coulombic potential. First, the eigenstates of potential (13) come in degenerate subsets, while this degeneracy - which prevents direct transitions between degenerate states - is lifted in the corresponding bottomonia. To allow such direct medium-induced transitions, we lift the degeneracy by hand, and give the states their vacuum masses [28].

Another issue is that not every known bottomonium has been assigned all its quantum numbers, and some states (e.g. in the $1 D$-quintuplet) have not yet been identified experimentally. To deal with these "missing states", we retain the degeneracy of states within $P$-wave triplets and $D$-wave quintuplets, even when they are differentiated experimentally.

Thirdly, while potential (13) admits an infinity of bound states, only a handful of bottomonia are actually stable against the strong interaction. And last, even though the scattering states of the Coulomb potential are known, yet we found it disturbing to use them to describe transition processes (dissociation or recombination) between bound bottomonia and free (anti)quarks, given that the latter do not exist in the vacuum. To cope with both these problems, we made a drastic assumption, namely that the bound eigenstates of potential (13) above and inclusive the $4 S$-level stand for unbound $b \bar{b}$ states. Additionally, we forbid by hand transitions from such "unbound" states back to bound ones.

The resulting spectroscopy of states we consider, with the transitions between them which we shall detail in Sect. IIIC are displayed in Fig. 2, Note that this slightly 


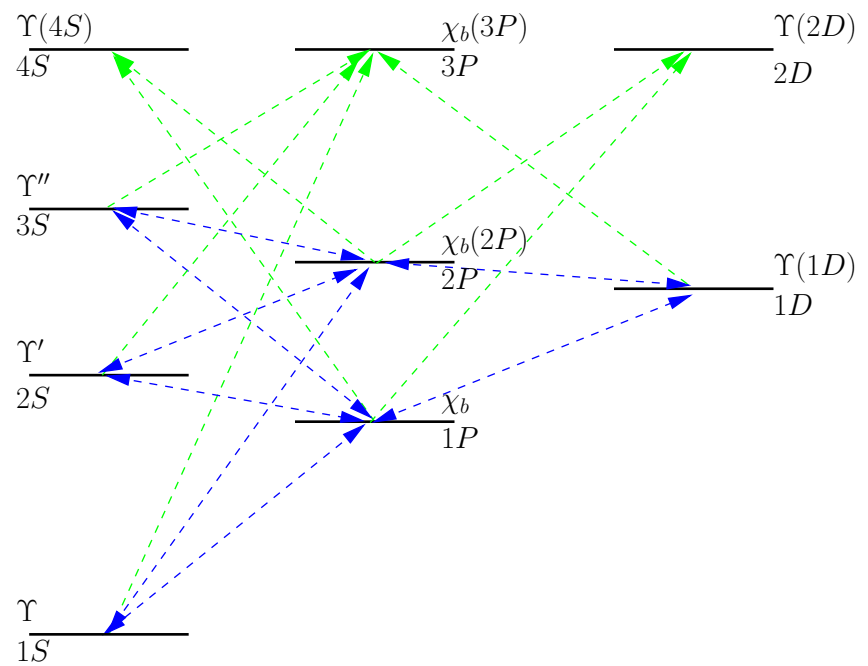

FIG. 2. Scheme of the spectroscopy of $b \bar{b}$ states and their transitions used in the calculations.

differs from the spectroscopy we used in 14], inasmuch as we have now added the $D$-wave states, which will impact our results due to their large overlap with the $P$-wave states. To estimate the error on our results, we also add one further level of (unbound) states, to which the bound levels can transition, but which cannot transition back.

\section{Quarkonium-plasma interaction}

Eventually, we need to specify the interaction between a $Q \bar{Q}$ pair and the gluon plasma. In this work we restrict ourselves to considering dipolar coupling, which induces vector transitions in the $Q \bar{Q}$ system 1 This amounts to considering the coupling of the $Q \bar{Q}$ pair to the dipolar part of the chromoelectric field of the gluons, which for an unpolarized plasma yields the interaction term

$V=-\mathbf{d} \cdot \mathbf{E}=-\mathrm{i} \sqrt{C_{F} \alpha_{s} \hbar c} \mathbf{r} \cdot \sum_{\lambda} \sqrt{\frac{2 \pi \hbar \omega_{\lambda}}{L^{3}}} \epsilon_{\lambda}\left(a_{\lambda}-a_{\lambda}^{\dagger}\right)$,

with $L$ the size of the box in which the chromoelectric field is quantized (which also appears in the normalization of the $b \bar{b}$ eigenstates) and $\boldsymbol{\epsilon}_{\lambda}$ the polarization vector

\footnotetext{
1 One might worry that single-gluon interactions induce transitions from color singlet to color octet $Q \bar{Q}$ states. While this is certainly true, yet it should be kept in mind that the model of quarkonia as pure bound $Q \bar{Q}$ states is only approximate: taking account the sea, an improved picture for a quarkonium is rather
}

$$
|(Q \bar{Q})\rangle=\psi_{Q \bar{Q}}|Q \bar{Q}\rangle+\psi_{Q \bar{Q} g}|Q \bar{Q} g\rangle+\psi_{Q \bar{Q} q \bar{q}}|Q \bar{Q} q \bar{q}\rangle+\cdots
$$

where the $Q \bar{Q}$ pair in $|Q \bar{Q} g\rangle,|Q \bar{Q} q \bar{q}\rangle \ldots$ can be in the octet representation, i.e. each quarkonium actually contains some admixture of color octet $Q \bar{Q}$. of gluon $\lambda$, while $\mathbf{d}$ (resp. r) denotes the dipole (resp. radius) operator for the $Q \bar{Q}$ pair.

Such an interaction term induces, to first order, transitions between $Q \bar{Q}$ states with different orbital quantum numbers only, as represented in Fig. 2,

Note that the dipolar coupling (14) actually rests on the assumption that both quark and antiquark in the pair see the same chromoelectric field. That is, we implicitly assume a large wavelength in computing the rates. While this holds for the gluons that induce transitions between bound states, yet it might not be granted for gluons that would dissociate the ground quarkonium statefor which one could instead use the rate computed in Ref. [25], which we did not do.

\section{RESULTS: EVOLUTION OF HEAVY QUARKONIA IN A THERMAL MEDIUM}

Within the framework of the model we have introduced in the previous section, we can now turn to solving the evolution equations for the populations of bottomonia in a thermal medium at temperature $T$. Following the same order as in Sect. III we first present results for the evolution of the internal degrees of freedom (Sect. IVA), then for the dynamics of the quarkonium center of mass (Sect. IV B). In Appendix B, we present results for the evolution in a Gaussian bath.

\section{A. Internal evolution of a static quarkonium}

Inspecting the set of equations (5) that govern the behavior of the populations when medium-induced emission and absorption processes are taken into account, a first result strikes the eye, even before solving the equations. Let the populations $\rho_{i i}^{Q \bar{Q}}$ be combined into a vector $\vec{\rho}$ and the system (5) be rewritten as

$$
\frac{\mathrm{d} \vec{\rho}}{\mathrm{d} t}(t)=\mathcal{U}_{\mathcal{R}} \vec{\rho}(t)
$$

with $\mathcal{U}_{\mathcal{R}}$ the time-evolution operator for the populations. In the vacuum, the matrix representation of $\mathcal{U}_{\mathcal{R}}$ in the basis of the energy eigenstates of the $Q \bar{Q}$ system (ordered in increasing energies) is triangular. This is no longer the case in the presence of a medium. Consequently, the eigenvalues and eigenstates of $\mathcal{U}_{\mathcal{R}}$ are not the same in a medium as in its absence 2 Physically this implies that the higher-energy $Q \bar{Q}$ states do not evolve independently from the more bound ones, as in the vacuum: because of medium-induced transitions, the former become coupled to the latter. As a consequence, past a transient regime, the populations of all states evolve with the same time scale.

\footnotetext{
${ }^{2}$ For a proof that $\mathcal{U}_{\mathcal{R}}$ is indeed diagonalizable, see Ref. [29].
} 


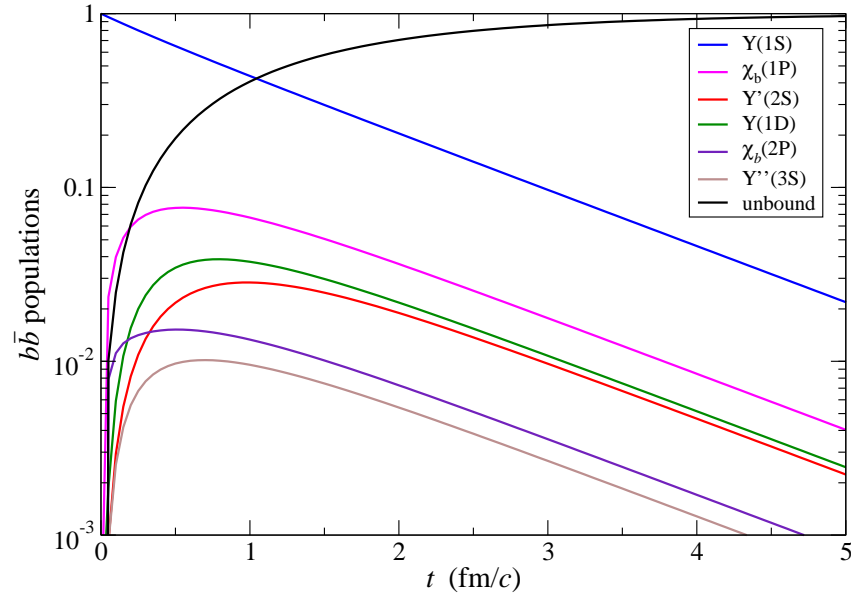

FIG. 3. Evolution of bottomonium populations in a thermal bath at $T=5 T_{c}$, with $T_{c}=170 \mathrm{MeV}$.

This holds irrespective of the assumed matrix elements between the $Q \bar{Q}$ states - provided every state is coupled to each other, at least indirectly. However, the value of the time scale is model-dependent and depends on the matrix elements and on the bath properties. In Fig. 3 we show the time evolution of $b \bar{b}$ states, modeled as in Sect. III, in a thermal bath at $T=5 T_{c}$, where the assumed initial condition consists of having all pairs in the ground state $\Upsilon(1 S)$ at $t=0$. The curves do not change significantly if we include one further level of unbound states (not shown). After the first fm/c or so, one reaches a quasi-equilibrated regime where the populations of all vacuum bound states decay with a characteristic time scale of $1.5 \mathrm{fm} / c$, while their ratios remain stationary 3 This result should be contrasted with the sequential-melting picture, where states would either be there, or totally melted according to the medium temperature, but cannot be regenerated through transitions from other states.

In a thermal bath at $2 T_{c}$, we find $8 \mathrm{fm} / c$ for the time scale of bottomonium evolution: as could be anticipated, the time scale decreases with rising temperature.

Focusing on the quasi-equilibrium population ratios, we show in Fig. 4 their dependence on the temperature of the plasma. These ratios differ significantly from their values for thermally equilibrated bottomonia, as would be expected in the framework of a statistical model [30]. This difference can easily be traced back to our forbidding transitions from unbound states to bound ones, so that the detailed balance condition

$$
\Gamma_{i \rightarrow k} \mathrm{e}^{-E_{i} / k_{B} T}=\Gamma_{k \rightarrow i} \mathrm{e}^{-E_{k} / k_{B} T} \quad \forall i, k,
$$

which guarantees the existence of an equilibrium with

\footnotetext{
3 The time scale reported in our previous work [14] was larger because there we had not considered the $D$-wave states.
}

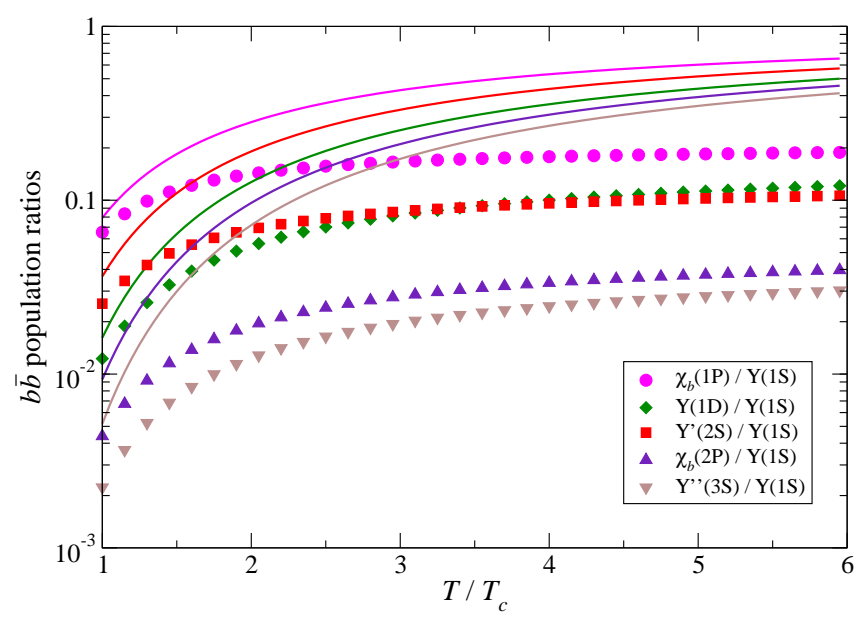

FIG. 4. Temperature dependence of the ratios of bottomonium populations. Symbols: quasi-equilibrium ratios within the master-equation formalism; full lines: ratios in a thermally equilibrated system.

populations proportional to the respective Boltzmann factors (see Sect. IIB), does not hold here.

One could argue that we have put this deviation from thermal equilibrium at long times by hand, by forbidding some of the emission transitions. This is true, but ultimately due to our over-simplified modeling of unbound states. As long as only a finite number of them is explicitly included, together with the back transitions, then equilibrium is reached after some finite time, which increases very rapidly with the number of states 4 When unbound states form a continuum, equilibrium is reached infinitely late, which is what we have modeled by setting some transition rates to 0 .

\section{B. Evolution of the external degrees of freedom}

Now that we have understood the internal dynamical evolution of the quarkonia, we can turn to investigating the evolution of the external degrees of freedom, and especially of the momentum distribution.

More precisely, we wish to consider the dynamics of the "momentum distribution of bound quarkonia" $\pi(\mathbf{p}, t)$, defined as the sum over all bound levels of the populations $\pi_{i, \mathbf{p}}(t) \equiv \rho_{i i, \mathbf{p p}}^{Q \bar{Q}}(t)$, where the density matrix is taken in the basis of the (vacuum) energy eigenstates. This distribution can evolve under the influence of two different effects induced by the plasma.

First, the $Q \bar{Q}$ bound state can be dissociated, i.e. it is "lost" from the populations that enter $\pi(\mathbf{p}, t)$, which thus decreases with time. More precisely (see Appendix A 2),

\footnotetext{
4 The attained equilibrium might differ from the thermal values when condition (15) is not fulfilled.
} 


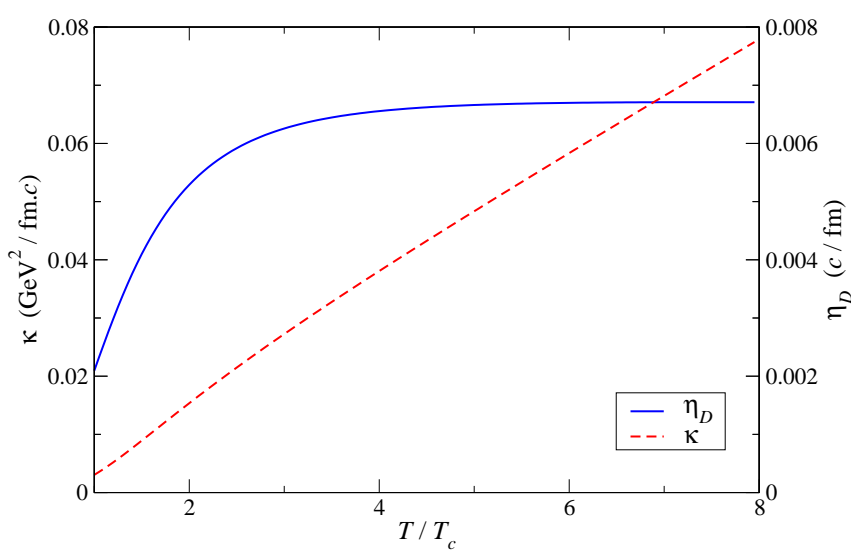

FIG. 5. Drift (full line) and diffusion (dashed line) coefficients of the Fokker-Planck equation (9) for the $b \bar{b}$ system immersed in a thermal bath at temperature $T$.

$\pi(\mathbf{p}, t)$ decays exponentially, with a rate that depends on p. The latter point is easily understandable, inasmuch as the energy of the gluons that interact with the moving $Q \bar{Q}$ pair is Doppler-shifted, so that pairs with different momentum do not see the same gluon spectrum.

On the other hand, the gluons can also induce internal transitions between bound states of the $Q \bar{Q}$ pair. In that case, the master-equation formalism predicts that, at least at small momentum $|\mathbf{p}|$ and for small momentum transfers $\hbar|\mathbf{k}|$, the rate of change of $\pi(\mathbf{p}, t)$ is significantly slower than the rates of the individual populations. Furthermore, in this regime the momentum distribution obeys the Fokker-Planck equation (9). We display in Fig. 5 the temperature dependence of the drift and diffusion coefficients in this equation, computed for the same bottomonium system as in Sect. IV A in a thermal bath 5 As can be checked, these coefficients satisfy the fluctuation-dissipation relation (10). While this hints at the equilibration of the external degrees of freedom of the bottomonia in a thermal plasma, yet one should keep in mind that the relevant time scale $\sim \eta_{D}^{-1}$ might actually be significantly larger than the time scale for bottomonium dissociation.

\section{DISCUSSION}

In this paper, we have applied the master-equation formalism to describe the evolution of heavy quarkonia in a gluon plasma, in complete analogy with the evolution of a small quantum system in contact with a reservoir. Independently of the model we used for the quarkonia, the plasma, and their interaction, several generic features emerge:

5 For such a bath, the condition on momentum underlying the derivation of the Fokker-Planck equation amounts to requiring that the bottomonia be non-relativistic.
1. When transitions between the various quarkonium states are allowed, then in the presence of a medium at fixed temperature, after a transient regime a stationary stage is reached, in which the populations of all states evolve together, as illustrated by Fig. 3 .

2. The ratios of these quasi-equilibrated populations in the stationary regime differ from the ratios in a statistical model for quarkonia in thermal equilibrium with the plasma, see Fig. 4.

3. The momentum distribution of bound quarkonia, considered irrespective of the internal state, satisfies a Fokker-Planck equation, at least in the nonrelativistic regime.

A further expected behavior - which we have not investigated in the present work, but manifests itself when studying the non-diagonal elements of the density matrix 23] - is that the interaction with the medium shifts the energy levels of the small system with respect to their vacuum values.

For the sake of illustration, we considered a simplified model for the heavy quarkonia, and more particularly for bottomonia rather than charmonia, and for the medium-induced transitions. Despite the rudimentary character of these models, the numerical values that come out for the characteristic time scale of the evolution of bottomonium populations and for the drift coefficient in the Fokker-Planck equation, including their dependence on temperature, are actually very similar to the values derived in more elaborate models for the $\Upsilon(1 S)$ lifetime [31] and for transport coefficients in the hardthermal-loop approach [32]. This is an encouraging finding, that shows the potential of the approach.

Here we wish to emphasize again that the starting point for the implementation of the formalism is either transition rates or, if one wants to start from scratch, a description of the quarkonia in vacuum and of the interaction with the medium. It is certainly tempting to use an in-medium quark-antiquark potential [33 38]. Yet one should not forget that such a potential already accounts for part of the coupling to the plasma, which then has to be subtracted out in a consistent manner from the other ingredients of the model, to avoid double counting. For instance, one can qualitatively expect that the screening of the potential in an in-medium potential picture corresponds to an increase of the transition rates from bound to unbound states in the formalism of the present paper.

Reformulating this differently, the master-equation formalism provides an evolution equation for the reduced density operator describing the quarkonia. Under the assumptions that make it local in time, this equation might be equivalent to a Heisenberg equation for $\rho^{Q \bar{Q}}$ involving a Hamiltonian with an effective potential, which incorporates the influence of the plasma, irrespective of whether the latter is in thermal equilibrium or not. Since we used a simplified vacuum quark-antiquark potential, we have not attempted to extract some corresponding effective 


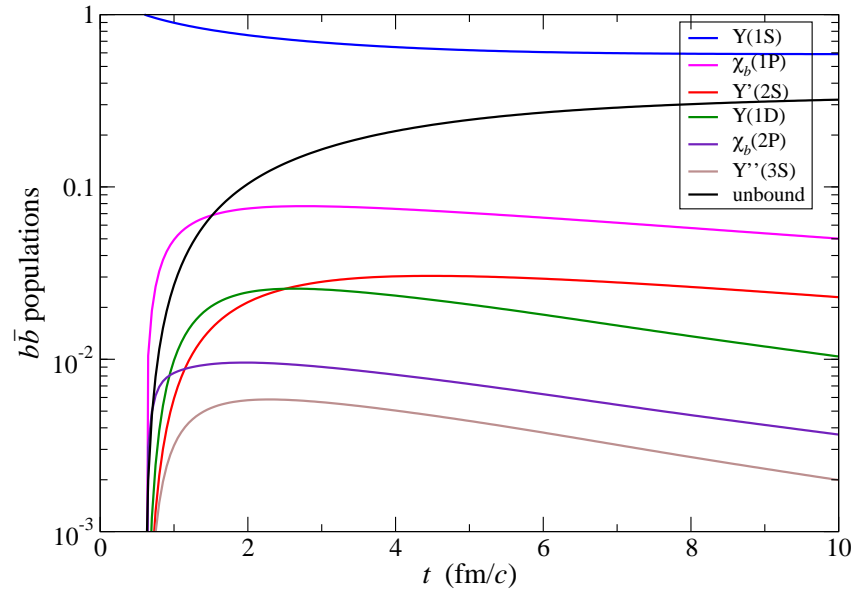

FIG. 6. Evolution of bottomonium populations in a thermal bath with evolving temperature.

potential6 Even then, it is clear that this in-medium potential would include an imaginary part, to account for the non-unitarity of the evolution of $\rho^{Q Q} \quad\left[\begin{array}{lll}6 & 11\end{array}\right]$.

For a future application to a more precise description of heavy quarkonia and their interaction with the fireball created in ultrarelativistic nucleus-nucleus collisions, one should discuss two aspects, namely the validity of the assumptions underlying the master-equation formalism and the relevance of the features listed above in the context of interest.

The main hypotheses behind the derivation of the master equation are twofold. First, the formalism holds provided the characteristic time scale of the medium fluctuations is much smaller than the time scale of the small system dynamics. This ensures that the possible correlations between medium and small system are continuously washed out, so that the evolution of the latter is Markovian. Given the size of the medium - equilibrating "parton" gas, quark-gluon plasma or hot hadron gascreated in high-energy heavy-ion collisions, this point is warranted. The second hypothesis, namely that of a "weak" coupling, which underlies the use of transition rates given by Fermi's golden rule, is actually less crucial. In a forthcoming study [15], we shall introduce an alternative approach releasing this assumption; however, the coupling strength does not affect the qualitative results summarized above.

Even though the master-equation approach seems to be applicable, at least as a good approximation, yet for the evolving medium created in high-energy heavy-ion collisions, some of those results have to be reexamined. Thus, the rate of evolution of the medium might be comparable to the rates of quarkonium-plasma interaction, and prevent the equilibration of the internal and exter-

6 This would amount to performing a Kraus decomposition [39] of the mapping from $\rho^{Q \bar{Q}}(0)$ to $\rho^{Q \bar{Q}}(t)$. nal degrees of freedom of the quarkonia. As an example, we present in Fig. 6 the evolution of the populations of bottomonia in a deconfined plasma whose temperature decreases with time as found at the center of the interaction region in hydrodynamical simulations of $\mathrm{Pb}-\mathrm{Pb}$ collisions at LHC energies [40]. One can see that the various bound states do not evolve similarly, so that the ratios of their populations do not remain constant, in contrast to point 1 above. Similarly, the description of the evolution of the momentum distribution of bound states through a Fokker-Planck equation, which relies on the equilibration of the internal degrees of freedom, does not hold either if the fireball cools down too rapidly.

This shows that results derived within a stationary picture for the quarkonia and/or the medium might actually not hold when real-time evolution is taken into account. The master-equation formalism, and other descriptions of dissipative quantum systems, can accommodate such a real-time evolution, since the assumptions made about the medium are rather minimal. Here we have shown that such an approach is possible, yet an accurate modeling of the dynamics of heavy quarkonia in ultrarelativistic nuclear collisions deserves further investigation.

\section{ACKNOWLEDGMENTS}

We thank Nirupam Dutta and Helmut Satz for helpful discussions and suggestions. C. G. acknowledges support form the Deutsche Forschungsgemeinschaft under grant GRK 881.

\section{Appendix A: Motion equation for quarkonia in a medium}

In this appendix, we detail the derivation of the Fokker-Planck equation that describes the evolution in a medium of the momentum distribution $\pi(\mathbf{p}, t)$ of bound quarkonium states.

As stated in Sect. III within our model one has to distinguish between transitions between bound $Q \bar{Q}$ states, which can take place in both directions, and transitions between a bound state and an unbound one, which can be dissociation processes only.

For the sake of brevity, we hereafter consider two different systems - coupled to reservoirs - with each two non-degenerate levels $E_{a}<E_{b}$. Let $\pi_{a, \mathbf{p}}, \pi_{b, \mathbf{p}}$ denote the diagonal elements of their respective reduced density matrices in the energy eigenstate basis. In the first system ("system I"), both levels correspond to "bound states" that can transition to each other. We then call "momentum distribution of the bound states" the sum $\pi^{\mathrm{I}}(\mathbf{p}, t) \equiv \pi_{a, \mathbf{p}}(t)+\pi_{b, \mathbf{p}}(t)$.

In opposition, for system II, only excitations from the lower to the higher level are allowed, while transitions back are forbidden, so that this constitutes an open system. The "momentum distribution of the bound states" 
is then $\pi^{\mathrm{II}}(\mathbf{p}, t) \equiv \pi_{a, \mathbf{p}}(t)$. Generalizing the calculation to more complicated spectroscopies is then straightfor- ward and amounts to combining the two behaviors that we encounter below.

\section{Evolution equations}

For those systems, the evolution equations (7) with the transition rates (8) read

$$
\begin{aligned}
& \frac{\mathrm{d} \pi_{a, \mathbf{p}}}{\mathrm{d} t}(t)=\sum_{\lambda} \frac{2 \pi}{\hbar^{2}}\left|\left\langle b ; 0|\tilde{V}| a ; 1_{\lambda}\right\rangle\right|^{2} \delta\left(\omega_{\lambda}-\omega_{b a}-\xi_{D}-\xi_{R}\right)\left[\eta\left(\left\langle n_{\lambda}\right\rangle+1\right) \pi_{b, \mathbf{p}+\hbar \mathbf{k}_{\lambda}}(t)-\left\langle n_{\lambda}\right\rangle \pi_{a, \mathbf{p}}(t)\right] \\
& \frac{\mathrm{d} \pi_{b, \mathbf{p}}}{\mathrm{d} t}(t)=\sum_{\lambda} \frac{2 \pi}{\hbar^{2}}\left|\left\langle b ; 0|\tilde{V}| a ; 1_{\lambda}\right\rangle\right|^{2} \delta\left(\omega_{\lambda}-\omega_{b a}-\xi_{D}+\xi_{R}\right)\left[\left\langle n_{\lambda}\right\rangle \pi_{a, \mathbf{p}-\hbar \mathbf{k}_{\lambda}}(t)-\eta\left(\left\langle n_{\lambda}\right\rangle+1\right) \pi_{b, \mathbf{p}}(t)\right]
\end{aligned}
$$

where $\omega_{b a}$ is the Bohr frequency of the transition, $\xi_{D}$ and $\xi_{R}$ are given by equation (8c), while $\eta=1$ for system I, $\eta=0$ for system II 7

Introducing the quantity

$$
\tilde{\Gamma}_{a b} \equiv \frac{2 \pi}{\hbar^{2}} \sum_{\lambda}\left|\left\langle b ; 0|\tilde{V}| a ; 1_{\lambda}\right\rangle\right|^{2} \delta\left(\omega_{\lambda}-\omega_{b a}\right)
$$

and a continuum representation, the evolution equations (A1) can be rewritten as

$$
\begin{aligned}
& \frac{\mathrm{d} \pi_{a, \mathbf{p}}}{\mathrm{d} t}(t)=\tilde{\Gamma}_{a b} \int_{0}^{\infty} \mathrm{d} \omega \frac{\omega^{3}}{\omega_{b a}^{3}} \int \frac{\mathrm{d} \Omega}{4 \pi} \delta\left(\omega-\omega_{b a}-\xi_{D}-\xi_{R}\right)\left(\eta[\langle n(\omega)\rangle+1] \pi_{b, \mathbf{p}+\hbar \mathbf{k}}(t)-\langle n(\omega)\rangle \pi_{a, \mathbf{p}}(t)\right), \\
& \frac{\mathrm{d} \pi_{b, \mathbf{p}}}{\mathrm{d} t}(t)=\tilde{\Gamma}_{a b} \int_{0}^{\infty} \mathrm{d} \omega \frac{\omega^{3}}{\omega_{b a}^{3}} \int \frac{\mathrm{d} \Omega}{4 \pi} \delta\left(\omega-\omega_{b a}-\xi_{D}+\xi_{R}\right)\left(\langle n(\omega)\rangle \pi_{a, \mathbf{p}-\hbar \mathbf{k}}(t)-\eta[\langle n(\omega)\rangle+1] \pi_{b, \mathbf{p}}(t)\right)
\end{aligned}
$$

where the modulus $|\mathbf{k}|=\omega / c$ is fixed by the respective Dirac distribution - noting that $\xi_{D}$ and $\xi_{R}$ actually also depend on $\omega-$, while $\mathrm{d} \Omega$ is the elementary solid angle around the direction in which $\mathbf{k}$ is pointing.

These equations clearly show that the motion of the small system center of mass shifts the frequency of the absorbed or emitted bath excitations away from the Bohr frequency. These processes also lead to an increase or a decrease of the momentum of the small system by $\hbar \mathbf{k}$.

\section{Open system: exact solution of the evolution equation}

When $\eta=0$, that is for system II, equations (A3) become simpler, and in particular the evolution of the population of the "bound state" decouples from that of the higher level. Equation (A3a), which describes the evolution of the bound-state momentum distribution $\pi^{\mathrm{II}}=\pi_{a}$, now reads

$$
\frac{\mathrm{d} \pi^{\mathrm{II}}}{\mathrm{d} t}(\mathbf{p}, t)=-\Gamma_{a \rightarrow b, \mathbf{p}} \pi^{\mathrm{II}}(\mathbf{p}, t),
$$

\footnotetext{
7 More generally, a factor $\eta \neq 1$ might account for non-equilibrated up and down transition rates.
}

with

$\Gamma_{a \rightarrow b, \mathbf{p}} \equiv \tilde{\Gamma}_{a b} \int_{0}^{\infty} \mathrm{d} \omega \frac{\omega^{3}}{\omega_{b a}^{3}}\langle n(\omega)\rangle \int \frac{\mathrm{d} \Omega}{4 \pi} \delta\left(\omega-\omega_{b a}-\xi_{D}-\xi_{R}\right)$,

where the dependence on $\mathbf{p}$ in the right-hand side is hidden in the Doppler frequency shift $\xi_{D}$. The solution to equation (A4) is trivial.

\section{Closed system: perturbative expansion of the evolution equation}

For $\eta \neq 0$, in particular for system I, further analytical progress with equations (A3) necessitates extra conditions on the size of the frequency shifts $\xi_{D}, \xi_{R}$ and of the momentum transferred in an absorption or emission process, namely $\xi_{D}, \xi_{R} \ll \Delta \omega$, the width of the reservoir spectrum, and $\hbar k \ll \Delta p$, the width of the momentum distribution. To ensure that these assumptions hold, it is sufficient that the two parameters

$$
\varepsilon_{1} \equiv \frac{\hbar k}{\Delta p} \quad \text { and } \quad \varepsilon_{2} \equiv \frac{\mathbf{k} \cdot \mathbf{p}}{M_{\mathcal{S}} \Delta \omega}
$$

be much smaller than unity, since this implies automatically $\xi_{R} / \Delta \omega \sim \varepsilon_{1} \varepsilon_{2} \ll 1$. 
Under these assumptions, we can Taylor-expand up to second order the Dirac distribution

$$
\begin{aligned}
\delta\left(\omega_{b a}-\omega+\xi_{D} \pm \xi_{R}\right) \simeq & \delta\left(\omega_{b a}-\omega\right)+\left(\xi_{D} \pm \xi_{R}\right) \delta^{\prime}\left(\omega_{b a}-\omega\right) \\
& +\frac{\left(\xi_{D} \pm \xi_{R}\right)^{2}}{2} \delta^{\prime \prime}\left(\omega_{b a}-\omega\right)
\end{aligned}
$$

and the momentum distributions $\pi_{a, \mathbf{p}}, \pi_{b, \mathbf{p}}$

$$
\pi_{\mathbf{p} \pm \hbar \mathbf{k}}(t) \simeq \pi_{\mathbf{p}}(t) \pm \hbar \mathbf{k} \cdot \nabla \pi_{\mathbf{p}}(t)+\frac{\hbar^{2}}{2} \sum_{i, j} k_{i} k_{j} \frac{\partial^{2} \pi_{\mathbf{p}}}{\partial p_{i} \partial p_{j}}(t)
$$

in equations (A3), and deduce simplified evolution equations for the bound-state momentum distribution $\pi^{\mathrm{I}}$ by identifying the factors of the various powers of $\varepsilon_{1}$ and $\varepsilon_{2}$.

The zeroth-order terms, which amount to neglecting the momentum transfer and the frequency shifts, are trivial and express the global conservation of the population of the system: $\mathrm{d} \pi^{\mathrm{I}}(\mathbf{p}) / \mathrm{d} t=0$. In turn, the linear terms in $\varepsilon_{1}, \varepsilon_{2}$ are automatically proportional to $\mathbf{k}$, and thus yield a vanishing contribution when averaged over all directions for $\mathbf{k}$.

At quadratic order, there are terms in $\varepsilon_{1}^{2}$-from the second-order term in expansion (A8) - and in $\varepsilon_{1} \varepsilon_{2}$ - from the term linear in $\xi_{R}$ in (A7) and from the product of the first-order terms of both expansions. The terms in $\varepsilon_{2}^{2}$ cancel out when summing equations (A3a) and (A3b). All in all, after performing the integrations by part necessary to get rid of the $\delta^{\prime}$ terms, followed by the straightforward integrations over $\omega$ and the direction of $\mathbf{k}$, one obtains

$$
\begin{aligned}
\frac{\mathrm{d} \pi^{\mathrm{I}}}{\mathrm{d} t}(\mathbf{p}, t)=\tilde{\Gamma}_{a b} & \frac{\hbar^{2} \omega_{b a}^{2}}{6 c^{2}}\left(\left[\left\langle n\left(\omega_{b a}\right)\right\rangle+1\right] \triangle \pi_{b, \mathbf{p}}(t)+\left\langle n\left(\omega_{b a}\right)\right\rangle \triangle \pi_{a, \mathbf{p}}(t)\right) \\
& -\tilde{\Gamma}_{a b} \frac{\hbar \omega_{b a}^{2}}{3 M_{\mathcal{S}} c^{2}} \frac{\mathrm{d}\langle n\rangle}{\mathrm{d} \omega}\left(\omega_{b a}\right) \nabla \cdot\left(\mathbf{p}\left[\pi_{a, \mathbf{p}}(t)-\pi_{b, \mathbf{p}}(t)\right]\right) \\
& +\tilde{\Gamma}_{a b} \frac{5 \hbar \omega_{b a}}{3 M_{\mathcal{S}} c^{2}} \boldsymbol{\nabla} \cdot\left[\mathbf{p}\left(\left[\left\langle n\left(\omega_{b a}\right)\right\rangle+1\right] \pi_{b, \mathbf{p}}(t)-\left\langle n\left(\omega_{b a}\right)\right\rangle \pi_{a, \mathbf{p}}(t)\right)\right] .
\end{aligned}
$$

The problem with this evolution equation is that it still contains the individual internal populations of the various internal states of the small system, and not only the total populations. An equation involving only the latter can be derived, provided the internal populations $\pi_{a, \mathbf{p}}, \pi_{b, \mathbf{p}}$ remain in fixed ratios, i.e. when the internal degrees of freedom are equilibrated.

Now, the evolution rates $\mathrm{d} \pi_{i, \mathbf{p}} / \mathrm{d} t$ themselves are of order 0 in $\varepsilon_{1}, \varepsilon_{2}$, much larger than the evolution rate for $\pi(\mathbf{p})$. That is, one may assume that the internal degrees of freedom reach stationary values on a much smaller time scale than the typical scale for the evolution of the momentum of the system. Inspecting equations (A3) with vanishing left-hand sides and considering only the leading terms in the Taylor expansions (A7) and (A8), one checks that the prescriptions

$$
\begin{aligned}
& \pi_{a, \mathbf{p}}(t)=\frac{1+\left\langle n\left(\omega_{b a}\right)\right\rangle}{1+2\left\langle n\left(\omega_{b a}\right)\right\rangle} \pi(\mathbf{p}, t) \\
& \pi_{b, \mathbf{p}}(t)=\frac{\left\langle n\left(\omega_{b a}\right)\right\rangle}{1+2\left\langle n\left(\omega_{b a}\right)\right\rangle} \pi(\mathbf{p}, t)
\end{aligned}
$$

are stationary solutions the evolution equations at leading order. Inserting them in equation (A9), one obtains

$$
\frac{\mathrm{d} \pi^{\mathrm{I}}}{\mathrm{d} t}(\mathbf{p}, t)=\kappa \Delta \pi^{\mathrm{I}}(\mathbf{p}, t)+\eta_{D} \boldsymbol{\nabla} \cdot\left[\mathbf{p} \pi^{\mathrm{I}}(\mathbf{p}, t)\right]
$$

where we have set

$$
\kappa \equiv \frac{\left[1+\left\langle n\left(\omega_{b a}\right)\right\rangle\right]\left\langle n\left(\omega_{b a}\right)\right\rangle}{1+2\left\langle n\left(\omega_{b a}\right)\right\rangle} \frac{\hbar^{2} \omega_{b a}^{2}}{3 c^{2}} \tilde{\Gamma}_{a b}
$$

and

$$
\eta_{D} \equiv-\frac{1}{1+2\left\langle n\left(\omega_{b a}\right)\right\rangle} \frac{\hbar \omega_{b a}^{2}}{3 M_{\mathcal{S}} c^{2}} \frac{\mathrm{d}\langle n\rangle}{\mathrm{d} \omega}\left(\omega_{b a}\right) \tilde{\Gamma}_{a b} .
$$

Equation A10a is an equation of the Fokker-Planck type, with constant diffusion coefficient $\kappa$ and drift coefficient $\eta_{D}$. Note that if one pushes the Taylor expansions to the next order, then the extra terms can be reexpressed as a momentum dependence of $\kappa$ and $\eta_{D}$.

In the case when the reservoir with which the small system is in contact is a thermal bath at temperature $T$, then $\langle n(\omega)\rangle$ is given by the Bose-Einstein distribution function, and one easily checks that the diffusion and drift coefficients (A10b)- (A10c) satisfy $\kappa=M_{\mathcal{S}} k_{B} T \eta_{D}$.

Let us end this appendix with some remarks on the assumptions underlying the derivation of equation (A10a). The Taylor expansion in powers of $\varepsilon_{1}$ and $\varepsilon_{2}$ is also the ingredient behind Landau's derivation of the FokkerPlanck equation from the Boltzmann equation without mean field term [41]. The smallness of $\varepsilon_{1}$ then amounts to considering that soft momentum exchanges with the bath play the major role in the evolution of the motion of the small system. Eventually, since the frequency shift due to the Doppler effect increases with the system momentum, the requirement of a small $\varepsilon_{2}$ implies that the description might not hold at high momentum. 


\section{Appendix B: Evolution of heavy quarkonia in a Gaussian bath}

In this appendix, we wish to take advantage of the fact that the master-equation formalism can accommodate various models of reservoirs, not only thermal baths, and demonstrate that our model of quarkonia in a gluon plasma naturally incorporates a behavior which is expected on physical grounds.

For that, we immerse bottomonia in the ground $\Upsilon(1 \mathrm{~S})$ state at $t=0$ in the Gaussian bath (12), keeping the same models for $b \bar{b}$ pairs and their interaction with the plasma as in Sects. IIIIV] As average energy $\bar{E}$ of the bath excitations, we consider first $\bar{E}=5 T_{c}$, then $10 T_{c}$, with a width $\Delta E=T_{c}$ in both cases. The resulting bottomonium populations, as a function of time, are shown in Fig. 7.

One finds two very different behaviors. For $\bar{E}=5 T_{c}$, the populations evolve similarly to the case of a thermal bath at $T=5 T_{c}$, see Fig. 6. On the other hand, for $\bar{E}=10 T_{c}$ there is almost no evolution over the same time interval. The physical interpretation of the latter finding is simple, namely that the gluons in the second bath are too energetic to view the bottomonia as a whole, and therefore cannot excite or dissociate efficiently, as was already found (for $J / \psi$ gluodissociation) in Ref. 26].
[1] T. Matsui and H. Satz, Phys. Lett. B 178 (1986) 416-422

[2] R. Rapp, D. Blaschke and P. Crochet, Prog. Part. Nucl. Phys. 65 (2010) 209-266 arXiv:0807.2470 [hep-ph]]

[3] L. Kluberg and H. Satz, in Relativistic Heavy-Ion Physics, Landolt-Börnstein New Series I/23A (Springer, 2009) arXiv:0901.3831 [hep-ph]]

[4] R. Rapp and H. van Hees, in Quark-Gluon Plasma 4, ed. by R. C. Hwa and X.-N. Wang (World Scientific, Singapore, 2010) pp. 111-206 [arXiv:0903.1096 [hep-ph]]

[5] N. Brambilla et al., Eur. Phys. J. C 71 (2011) 1534 arXiv:1010.5827 [hep-ph]]

[6] M. Laine, O. Philipsen, P. Romatschke and M. Tassler, JHEP 0703 (2007) 054 hep-ph/0611300

[7] A. Beraudo, J.-P. Blaizot and C. Ratti, Nucl. Phys. A 806 (2008) 312 arXiv:0712.4394 [nucl-th]]

[8] M. Laine, JHEP 0705 (2007) 028 arXiv:0704.1720 [hep$\mathrm{ph}]]$

[9] M. A. Escobedo and J. Soto, Phys. Rev. A 78 (2008) 032520 arXiv:0804.0691 [hep-ph]]

[10] N. Brambilla, J. Ghiglieri, A. Vairo and P. Petreczky, Phys. Rev. D 78 (2008) 014017 arXiv:0804.0993 [hepph]]

[11] A. Rothkopf, T. Hatsuda and S. Sasaki, Phys. Rev. Lett. 108 (2012) 162001 arXiv:1108.1579 [hep-lat]]

[12] U. Weiss, Quantum dissipative systems, 3rd edn. (World Scientific, Singapore, 2007)

[13] C. Young and K. Dusling, arXiv:1001.0935 [nucl-th]

[14] N. Borghini and C. Gombeaud, arXiv:1103.2945 [hep-ph]

[15] N. Dutta, N. Borghini, in preparation

[16] D. Levin-Plotnik and B. Svetitsky, Phys. Rev. D 52 (1995) 4248-4250 hep-ph/9503305

[17] A. Polleri, T. Renk, R. Schneider and W. Weise, Phys. Rev. C 70 (2004) 044906 nucl-th/0306025

[18] L. Yan, P. Zhuang and N. Xu, Phys. Rev. Lett. 97 (2006) 232301 nucl-th/0608010

[19] B. K. Patra and V. J. Menon, Nucl. Phys. A 708 (2002) 353-364 hep-ph/0112196

[20] C. Young and E. Shuryak, Phys. Rev. C 79 (2009) 034907 arXiv:0803.2866 [nucl-th]]

[21] L. Grandchamp, R. Rapp and G. E. Brown, Phys. Rev. Lett. 92 (2004) 212301 hep-ph/0306077

[22] X. Zhao and R. Rapp, Phys. Rev. C 82 (2010) 064905 arXiv:1008.5328 [hep-ph]]
[23] C. Cohen-Tannoudji, J. Dupont-Roc and G. Grynberg, Atom-Photon Interactions (John Wiley \& Sons, New York, 1998) Chapter IV

[24] E. Eichten, S. Godfrey, H. Mahlke and J. L. Rosner, Rev. Mod. Phys. 80 (2008) 1161-1193 hep-ph/0701208

[25] D. Kharzeev and H. Satz, in Quark-Gluon Plasma 2, ed. by R. C. Hwa (World Scientific, Singapore, 1995) pp. 395-453 hep-ph/9505345

[26] X.-M. Xu, D. Kharzeev, H. Satz, X.-N. Wang, Phys. Rev. C 53 (1996) 3051-3056 hep-ph/9511331

[27] R. L. Thews, M. Schroedter and J. Rafelski, Phys. Rev. C 63 (2001) 054905 hep-ph/0007323

[28] K. Nakamura et al. (Particle Data Group), J. Phys. G 37 (2010) 075021.

[29] E. B. Davies, Commun. Math. Phys. 39 (1974) 91-110

[30] P. Braun-Munzinger and J. Stachel, Phys. Lett. B 490 (2000) 196-202 nucl-th/0007059

[31] L. Grandchamp, S. Lumpkins, D. Sun, H. van Hees and R. Rapp, Phys. Rev. C 73 (2006) 064906 hep-ph/0507314

[32] A. Beraudo, A. De Pace, W. M. Alberico and A. Molinari, Nucl. Phys. A 831 (2009) 59-90 arXiv:0902.0741 [hep$\mathrm{ph}]$ ]

[33] S. Digal, P. Petreczky and H. Satz, Phys. Lett. B 514 (2001) 57-62 hep-ph/0105234

[34] C. Y. Wong, Phys. Rev. C 72 (2005) 034906 hep-ph/0408020

[35] F. Arleo, J. Cugnon and Y. Kalinovsky, Phys. Lett. B 614 (2005) 44-52 hep-ph/0410295.

[36] W. M. Alberico, A. Beraudo, A. De Pace and A. Molinari, Phys. Rev. D 75 (2007) 074009 hep-ph/0612062

[37] D. Cabrera and R. Rapp, Phys. Rev. D 76 (2007) 114506 hep-ph/0611134

[38] A. Mócsy and P. Petreczky, Phys. Rev. D 77 (2008) 014501 arXiv:0705.2559 [hep-ph]]

[39] K. Kraus, States, Effects, and Operations, Lecture Notes in Physics 190 (Springer, 1983)

[40] C. Shen, U. W. Heinz, P. Huovinen and H. Song, Phys. Rev. C 84 (2011) 044903 arXiv:1105.3226 [nucl-th]]

[41] E. M. Lifshitz and L. P. Pitaevskii, Physical Kinetics (Pergamon, Oxford, 1981) §21 

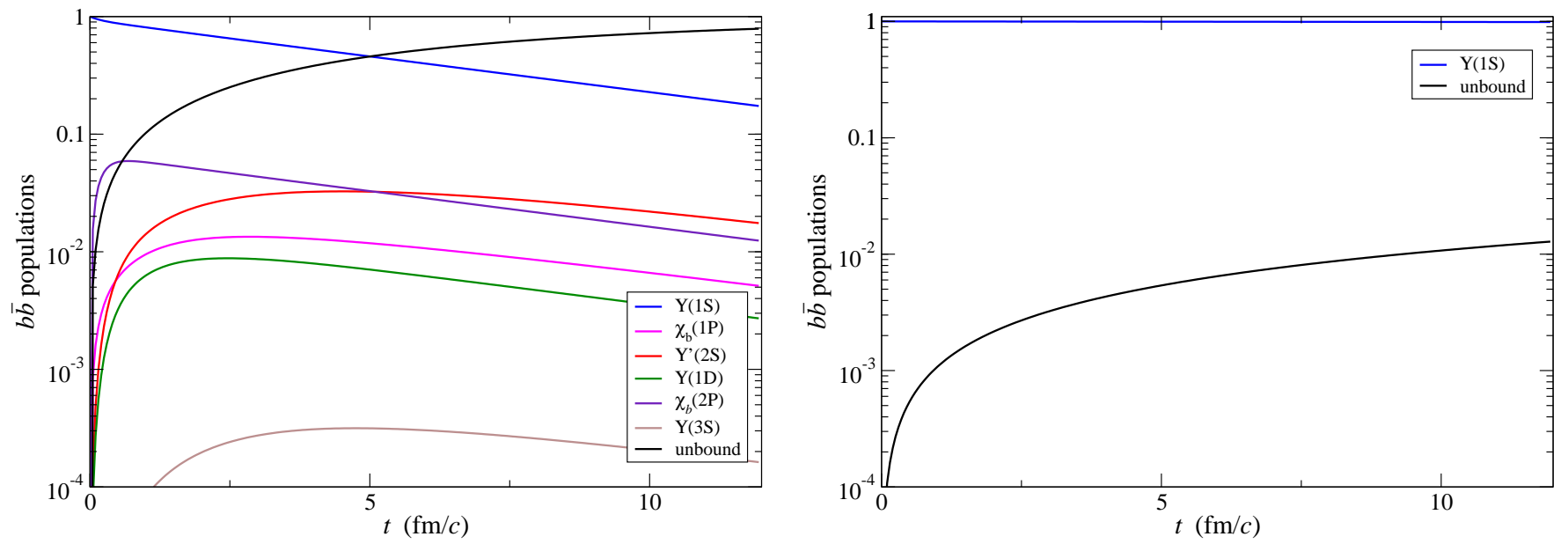

FIG. 7. Evolution of bottomonium populations in a Gaussian bath. Left: peaked around $5 T_{c}$; Right: peaked around $10 T_{c}$. 